EPJ Web of Conferences 49, 15015 (2013)

DOI: $10.1051 /$ epjconf/20134915015

(C) Owned by the authors, published by EDP Sciences, 2013

\title{
Search for SUSY in final states with photons at CMS
}

\author{
Eleni Ntomari ${ }^{1,2}$, a , On behalf of the CMS Collaboration \\ ${ }^{1}$ NCSR Demokritos, Athens, Greece \\ ${ }^{2}$ National Technical University of Athens, Greece
}

\begin{abstract}
.
The Compact Muon Solenoid (CMS) collaboration has developed a complete program of searches beyond the Standard Model (SM) covering a wide range of final states. This document focuses on searches in final states with photons and missing transverse energy ( $\mathrm{E}_{\mathrm{T}}^{\text {miss }}$ ) organised in three analyses. The first two include comparison of the $\mathrm{E}_{\mathrm{T}}^{\mathrm{miss}}$ distribution (isolation sideband method) in events with either at least two photons plus at least one hadronic jet, or at least one photon plus at least two hadronic jets. The third analysis corresponds to a new approach, the Jet-Gamma Balance (JGB) method, for events with at least one photon plus at least three hadronic jets. We observe no significant deviations from the SM expectation and thus derive upper limits on the signal cross section at the $95 \%$ confidence level (CL) for a range of squark, gluino and neutralino mass points in the Gauge Mediated Supersymmetry Breaking scenario.
\end{abstract}

\section{Introduction}

Supersymmetry (SUSY) is one of the most appealing extensions to the Standard Model, solving the hierarchy problem and providing an explanation for dark matter. It introduces a symmetry between fermions and bosons resulting in a superpartner (sparticle) for each SM particle with identical quantum numbers except spin. Since no sparticles have been observed yet, SUSY (if it exists) must be a broken symmetry, allowing the sparticles to be much heavier than their SM partners. The version of supersymmetry based on General Gauge-Mediated (GGM) SUSY breaking [2-4] is of particular theoretical interest for physics beyond the Standard Model as it not only stabilizes the mass of the SM Higgs boson and drives the grand unification of forces, but also avoids the large flavor-changing neutral currents that trouble other SUSYbreaking scenarios. According to the GGM SUSY breaking scenario, the gravitino $(\tilde{G})$ is the lowest mass SUSY particle (LSP) and the lightest neutralino $\left(\tilde{\chi_{1}^{0}}\right)$ is the nextto-lowest mass SUSY particle (NLSP). In the documented analyses, the neutralino consists of either the bino; superpartner of the U(1) gauge field, or the wino; superpartner of the SU(2) gauge field. A bino-like neutralino decays predominantly into a gravitino and a photon with a branching fraction $\sim \cos ^{2} \theta_{\mathrm{w}}$, while the decay to a gravitino and a $\mathrm{Z}$ boson is sub-dominant $\left(\sim \sin ^{2} \theta_{\mathrm{w}}\right)$. In the case of a wino-like neutralino, the splitting between the charged and the neutral wino is in general small. Thus the neutral and charged winos become co-NLSPs, with the charged winos decaying directly into the gravitino and a $W^{ \pm}$as well, while the neutral decaying dominantly to a

\footnotetext{
a. e-mail: Eleni.Ntomari@ cern.ch
}

gravitino and a $\mathrm{Z}$ boson $\left(\backsim \cos ^{2} \theta_{\mathrm{w}}\right)$ and sub-dominantly to a gravitino and a photon $\left(\backsim \sin ^{2} \theta_{\mathrm{w}}\right)$.

We only consider cases in which at least one of the neutralinos decays promptly to a gravitino and a photon. This is an experimentally accessible channel since photons can be identified with high efficiency and purity at hadron collider experiments. Since the gravitino escapes detection, it leads to missing transverse energy in the event. Assuming R-parity conservation, SUSY particles are pair produced. Those particles are dominantly strongly interacting with decay chains including one or several quarks/gluons. Therefore, events with a pure binolike neutralino are expected to contain two photons and two gravitinos plus additional Standard Model particles in the final states and thus motivating di-photon analyses. The created SM particles are either a photon or a $\mathrm{Z}$ or a Higgs boson, if the mass difference between the NLSP and the LSP allows it kinematically. A second signature, with just one photon, is possible if one of the NLSPs decays into a $\mathrm{Z}$ boson instead of a photon. Lastly in the case of a wino-like neutralino, the di-photon final state is quite suppressed, but single-photon final states may be significant.

We present three analyses. The first two [7] include searches using the $\mathrm{E}_{\mathrm{T}}^{\mathrm{miss}}$ template method, performed with data collected by the CMS detector [1] at $\sqrt{s}=8 \mathrm{TeV}$ produced at the Large Hadron Collider (LHC), corresponding to an integrated luminosity of $4.04 \mathrm{fb}^{-1}$, on events with either at least two photons plus at least one hadronic jet, or at least one photon plus at least two hadronic jets. The third one corresponds to data collected at $\sqrt{s}=7 \mathrm{TeV}$ with an integrated luminosity of $4.7 \mathrm{fb}^{-1}$, for events with at least one photon plus at least three hadronic jets, analysed with a new approach, the Jet-Gamma Balance method [8]. 


\section{Data Sample and Event Selection}

The data used in these analyses are accumulated using the CMS two-level trigger system requiring the presence of at least two photons or at least one high-energy photon and significant hadronic activity. The offline cuts are more restrictive than the trigger requirements, to ensure good understanding of the efficiency. In the following paragraphs we briefly describe the physics object selection.

The photon candidates are reconstructed from clusters of energy in the CMS electromagnetic crystal calorimeter (ECAL). The ECAL was designed to have both excellent energy resolution and high granularity, making it a powerful instrument to measure photons with high precision at the LHC. Photon objects are created based on depositions of energy within the ECAL. Because of the material in front of the calorimeter, photon conversions and bremsstrahlung from electrons and positrons is likely. The strong magnetic field often leads the energy flow associated with primary electrons or converted primary photons to spread in $\phi$. To collect the photon energy in the ECAL, local deposits are summed into superclusters that are extended in $\phi$. A supercluster passing a list of identification and quality criteria (e.g. shower shape) is promoted to a photon as described in [11]. In addition, the absence of pixel tracker hits matching to the supercluster is required to descriminate photons from electrons. To increase the purity of the photon sample, isolation requirements are applied using combined information from ECAL, HCAL and the tracker. In total, not more than $6 \mathrm{GeV}$ pile-up subtracted [12] energy deposit is allowed within a cone of $\Delta R=\sqrt{\Delta \phi^{2}+\Delta \eta^{2}}=0.3$.

The definition of an electron, as already discussed, is similar to a photon except for the requirement of hits on the pixel tracker matching to the supercluster. Electrons are used to estimate the backgrounds from processes with an electron misidentified as a photon and real $\mathrm{E}_{\mathrm{T}}^{\mathrm{miss}}$ (e.g. $W \rightarrow e v$ ) similarly to [7] and references within.

Jets are clustered with the anti- $\mathrm{k}_{T}$ clustering algorithm [13] with a size parameter $R=0.5$ and reconstructed with the Particle-Flow reconstruction algorithm [9]. Energy scale corrections are applied in the jet to take into account the non-linear jet energy response [10]. To prevent double counting, jets must be isolated from photons by $\Delta R>0.4$. For the $\mathrm{H}_{\mathrm{T}}$ offline selection (applied on the single photon analyses), calorimeter jets (jets reconstructed using only the energy deposits in the calorimeter towers) are used in order to follow the online selection. Within the Particle-Flow framework the $\mathrm{E}_{\mathrm{T}}^{\mathrm{miss}}$ is the $\mathrm{p}_{\mathrm{T}}$ of the negative vector sum of all reconstructed particles [9].

\section{Background Estimation Methodology}

This signature of SUSY signal events can be mimicked by various SM backgrounds. The main backgrounds arise from SM processes with misidentified photons and/or mismeasured $E_{\mathrm{T}}^{\mathrm{miss}}$. The dominant contribution comes from QCD processes such as direct di-photon, photon plus jets, and multijet production. The strategy for determining this background is to use the $\mathrm{E}_{\mathrm{T}}^{\mathrm{miss}}$ distribution of control samples, that are kinematically similar to the candidate sample, while having no true $\mathrm{E}_{\mathrm{T}}^{\mathrm{miss}}$ [7]. This background sample is selected by applying the signal selection requirements, except that the photon candidate is required to fail the photon identification criteria but to satisfy a loose isolation requirement, and it is weighted to correct for the difference in $\mathrm{p}_{\mathrm{T}}$ spectra of misidentified and genuine photons. Additionally or alternatively, we can use the JGB method [8]. The JGB variable measures the imbalance between the photon $\mathrm{p}_{\mathrm{T}}$ and the $\mathrm{p}_{\mathrm{T}}$ of hadronic recoil, and it is defined as : $J G B=\left|\sum \overrightarrow{p_{T}^{\text {jets }}}\right|-\left|\overrightarrow{p_{T}^{\gamma}}\right|=\left|\overrightarrow{E_{T}^{\text {miss }}}+\overrightarrow{p_{T}^{\gamma}}\right|-\left|\overrightarrow{p_{T}^{\gamma}}\right|$. A high positive value of JGB favors events with a photon produced at the end of a decay chain (e.g. SUSY cascade decay). Photons produced closer to the primary interaction are kinematically balanced and tend to produce JGB close to zero. SM backgrounds with only mismeasured $\mathrm{E}_{\mathrm{T}}^{\text {miss }}$ are evenly poppulating JGB $>0$ and JGB $<0$ regions. Thus the region with JGB $<0$ can be used as a control sample to model the background event yield expected in JGB $>0$.

The second kind of background comes from processes with real $\mathrm{E}_{\mathrm{T}}^{\mathrm{miss}}$. It is dominated by $\mathrm{W} \gamma$ and $\mathrm{W}$ plus jets events, where the $\mathrm{W}$ decays into an electron plus a neutrino, with the electron or jet misidentified as a photon and the neutrino leading to $\mathrm{E}_{\mathrm{T}}^{\mathrm{miss}}$. Since the photon is expected to behave similarly to an electron in the ECAL, electrons can be misidentified as photons (except that they have hits matching the particle track in the pixel detector). Measuring the electron-photon misidentification rate $\mathrm{f}_{e->\gamma}$, we can determine the contribution of this background by applying $\mathrm{f}_{e->\gamma}$ to the $\mathrm{E}_{\mathrm{T}}^{\text {miss }}$ (JGB) distribution.

Additional backgrounds can contribute due to initialstate (ISR) and final-state radiation (FSR) of photons. Both ISR and FSR, in events with electrons in the final state, are already covered by the EWK background prediction from data. The remaining contributions from $\mathrm{W}, \mathrm{Z}$, and $\mathrm{t} \overline{\mathrm{t}}$ events are taken from MC simulation. More details about the additional backgrounds and how they are handled by each one of the analyses, as well as description of each method's systematic uncertainties and the exact event selection criteria, can be found in Ref.[7] and Ref.[8].

\section{Di-photon $\mathrm{E}_{\mathrm{T}}^{\text {miss }}$ based analysis at $\sqrt{s}=8 \mathrm{TeV}$}

The $\mathrm{E}_{\mathrm{T}}^{\mathrm{miss}}$ distribution in the $\gamma \gamma$ sample, for events with at least one hadronic jet, is presented in Fig. 1 as points with error bars. The red hatched area indicate the total background uncertainties. The blue shaded area represents the estimated amount of the EWK background, while the grey shaded area corresponds to the QCD background. The goal of these analyses is to find evidence for the production of New Physics by observing an excess of events above the $\mathrm{SM}$ background in the high $\mathrm{E}_{\mathrm{T}}^{\text {miss }}$ (JGB) region. Since no excess is observed, upper limits are derived for the GMSB scenario.

The SUSY signal events in the analyses presented here, are generated with the PYTHIA event generator. 


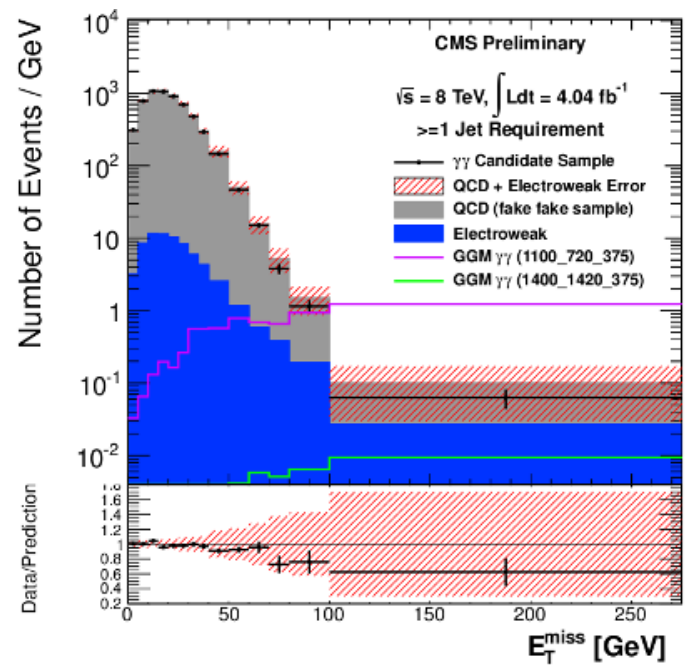

Figure 1. $\mathrm{E}_{\mathrm{T}}^{\mathrm{miss}}$ spectrum of $\gamma \gamma$ data compared to QCD prediction together with the small EWK background for events with at least one jet. The red hatched areas indicate the total background uncertainties. Two example GGM points on either side of the exclusion boundary $\left(m_{\tilde{q}} / m_{\tilde{g}} / m_{\tilde{\chi}_{1}^{0}}\right.$ in $\left.\mathrm{GeV}\right)$ are also shown.

In particular SUSY GGM events are generated in a twodimensional grid of the gluino and squark masses for a fixed neutralino mass of $375 \mathrm{GeV}$ in the benchmark models in [14]. A signal point is excluded if the expected cross section exceeds our upper limit. In order to achieve good signal sensitivity, the limits were calculated in six distinct $\mathrm{E}_{\mathrm{T}}^{\text {miss }}$ bins in $\mathrm{GeV}$ : $[50,60),[60,80),[80,100),[100,140)$, $[140,180),[180, \infty)$ and the multi-channel counting experiments are combined into one, taking into account bin-tobin correlations of the systematic uncertainties, as well as the significance of each bin. The calculated 95\% CL exclusion contours for a bino- and wino-like neutranino are shown in Fig. 2 and Fig. 3 respectively.

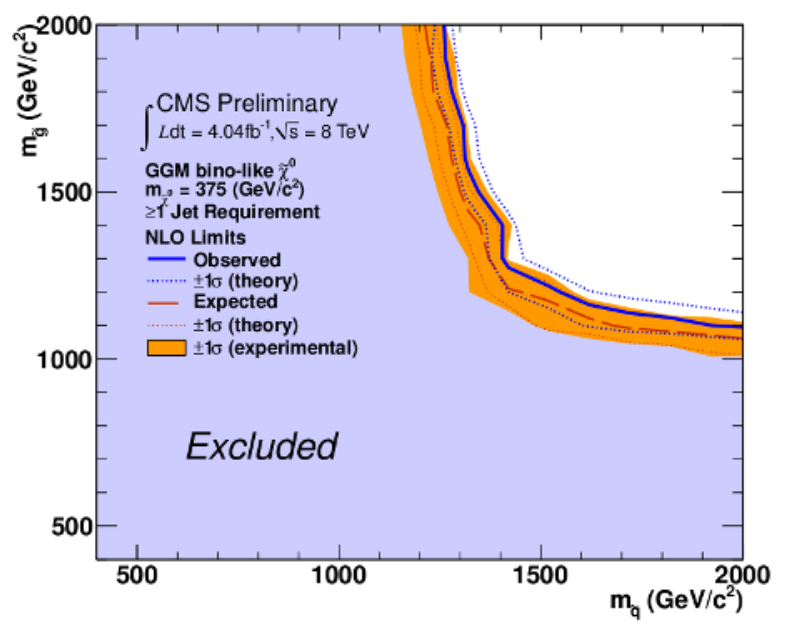

Figure 2. Calculated 95\% CL exclusion contours for a bino-like neutralino in the squark-gluino mass plane for the di-photon analysis with the isolation sideband method.

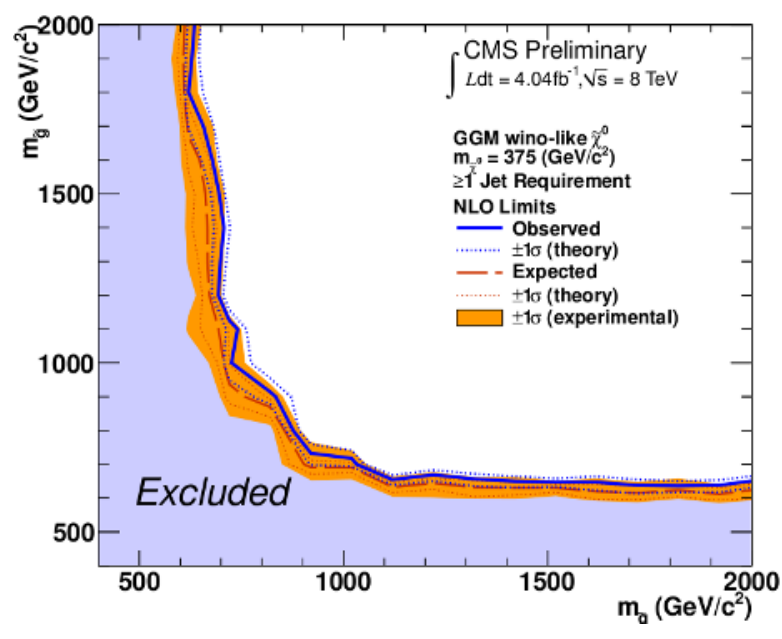

Figure 3. Calculated 95\% CL exclusion contours for a wino-like neutralino in the squark-gluino mass plane for the di-photon analysis with the isolation sideband method.

\section{Single photon $\mathrm{E}_{\mathrm{T}}^{\text {miss }}$ based analysis at $\sqrt{s}=8 \mathrm{TeV}$}

In the single photon analyses we have analogous results. The $\mathrm{E}_{\mathrm{T}}^{\mathrm{miss}}$ distribution for all background components, together with the data ilustrated as points with error bars, are shown in Fig. 4. Superimposed there are two GGM benchmark signal samples, one excluded (red line) and one not excluded (violet line) by this analysis. No excess beyond the SM prediction is observed and thus we proceed to limits setting.

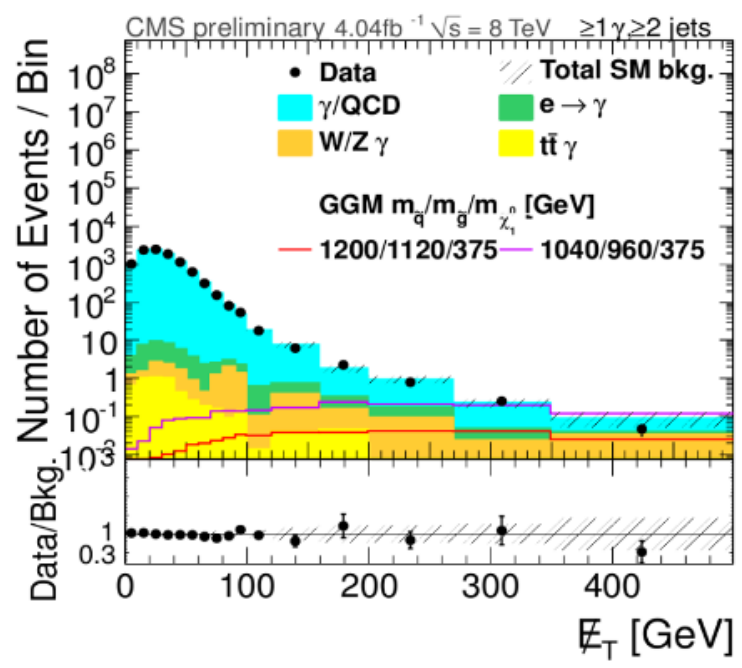

Figure 4. Total standard model background prediction compared to the number of single-photon events, including two GGM benchmark signal benchmark points as examples.

For this analysis six distinct $\mathrm{E}_{\mathrm{T}}^{\text {miss }}$ bins are used for the limit calculation : $[100,120),[120,160),[160,200)$, 
$[200,270),[270,350)$, and $[350, \infty)$ given in GeV. The relevant limits are illustrated in Fig. 5 and Fig. 6 for the binoand wino-like neutralinos.

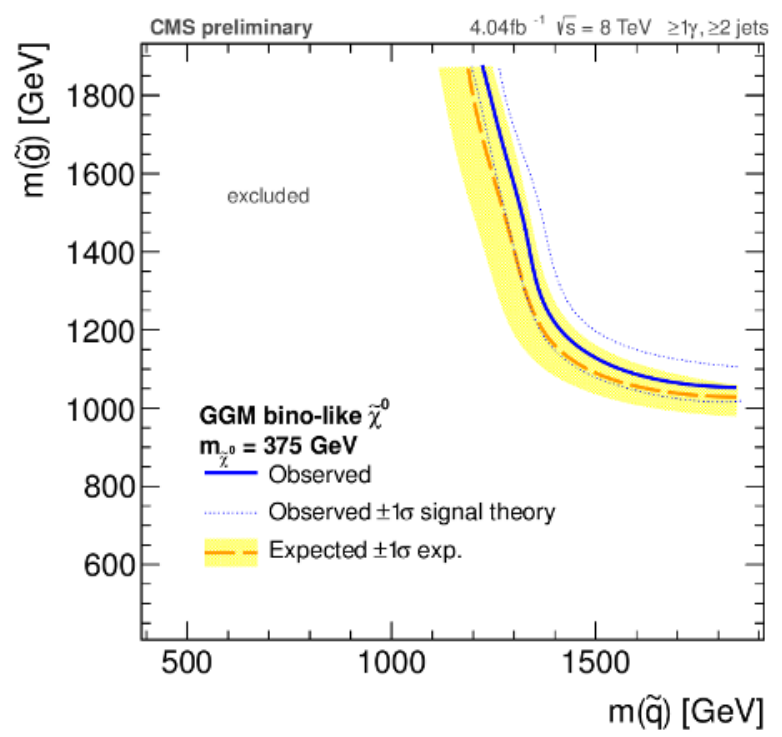

Figure 5. Calculated 95\% CL exclusion contours for a bino-like neutralino in the squark-gluino mass plane for the single-photon analysis with the isolation sideband method.

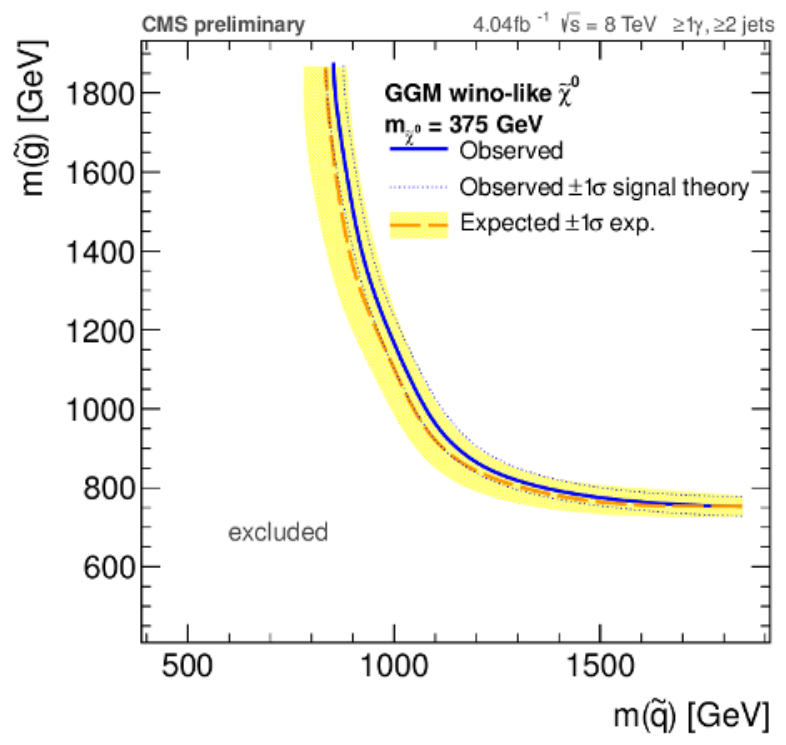

Figure 6. Calculated 95\% CL exclusion contours for a wino-like neutralino in the squark-gluino mass plane for the single-photon analysis with the isolation sideband method.

\section{Single photon JGB based analysis at $\sqrt{s}=7 \mathrm{TeV}$}

The goal of this analysis is to find evidence for the production of new physics by observing an excess of events above the Standard Model background in the high JGB region. Figure 7, shows the comparison between background prediction and observed events in the JGB $>0$ region. The error bars indicate the statistical uncertainties in data only, while the dashed band corresponds to the total background uncertainties which include bin by bin correlations. Taking into considerartion the errors, no excess is observed since the data are in satisfactory agreement with the standard model expectation. Hence upper limits are derived on potential signals of new physics models (GMSB).

In the JGB based analysis, the limits were calculated for three distinct JGB bins in GeV : [80,100), [100,120), $[120, \infty)$. The relevant limits, along with the corresponding to the $\mathrm{E}_{\mathrm{T}}^{\mathrm{miss}}$ based analysis (SUS-12-001, [15]), are illustrated in Fig. 8 and Fig. 9 for the bino- and wino-like scenarios.

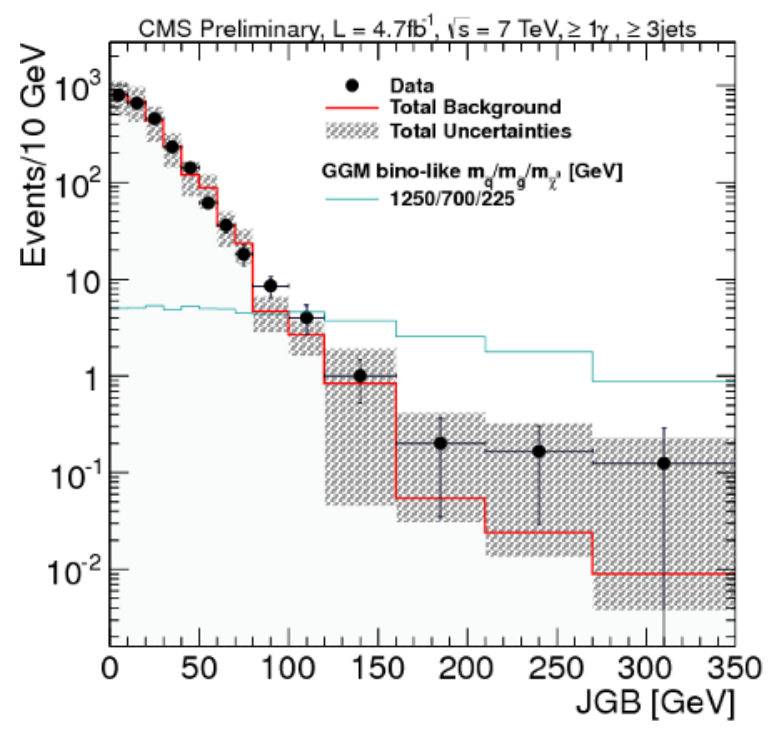

Figure 7. Comparison between the observed data and the predicted background event yield in the signal region for the singlephoton analysis (JGB method). The error bars indicate the statistical uncertainties in data only, while the dashed band corresponds to the total background uncertainties. An example benchmark GGM signal $\left(m_{\tilde{q}} / m_{\tilde{g}} / m_{\tilde{\chi}_{1}^{0}}\right.$ in $\left.\mathrm{GeV}\right)$ is also overlaid.

\section{Conclusions}

To summarize, we have searched for evidence of GGM SUSY production in diphoton and single-photon final states using the $\mathrm{E}_{\mathrm{T}}^{\mathrm{miss}}$ spectrum based on $2012 \mathrm{CMS}$ data comprising $4.04 \mathrm{fb}^{-1}$ of pp collisions at $\sqrt{s}=8 \mathrm{TeV}$ and in single-photon final states using the JGB spectrum for 2011 CMS data at $\sqrt{s}=7 \mathrm{TeV}$ for an integrated luminosity $4.7 \mathrm{fb}^{-1}$. In all the cases the observation is in good aggrement with the data driven predictions, and upper limits (95\% CL) are set in the GGM SUSY parameter space of squark and gluino masses for the bino- and wino-like scanarios. As it can be seen from the relevant limit plots, the di-photon analyses sets more strict limits to the binolike scenario, while the single photon analyses set tighter 


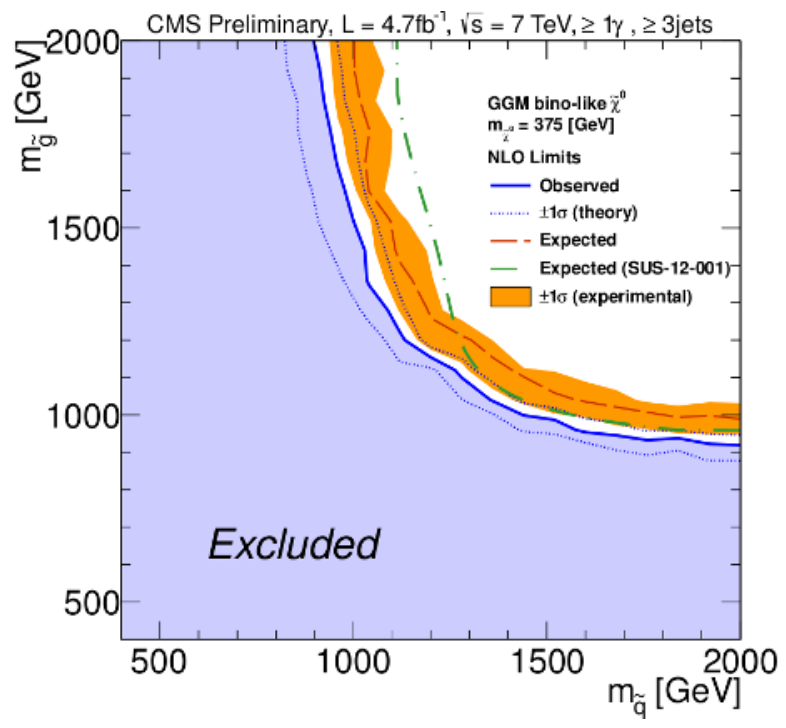

Figure 8. Calculated 95\% CL exclusion contours for a bino-like neutralino in the squark-gluino mass plane for the single photon analysis with the JGB method.

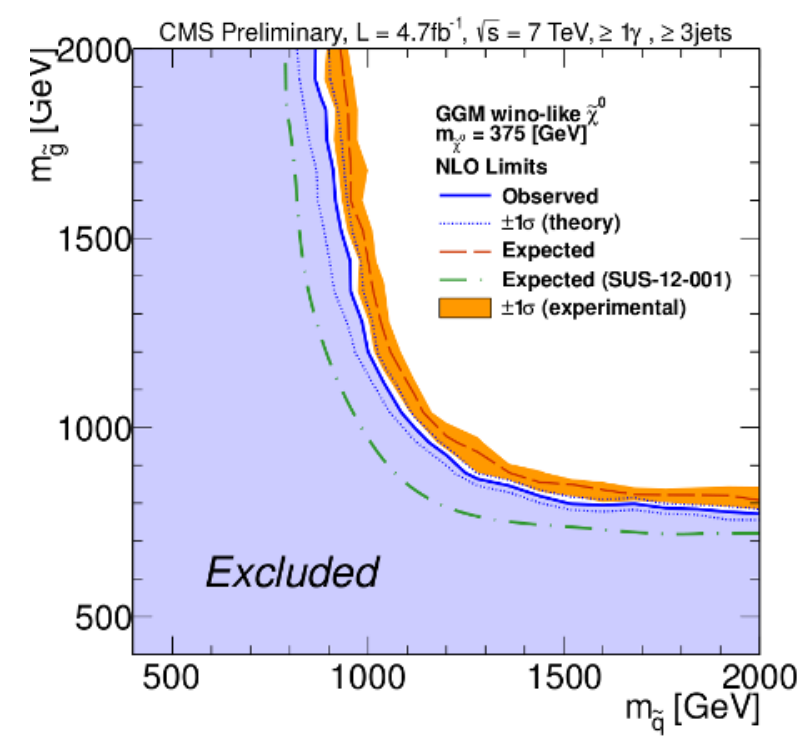

Figure 9. Calculated 95\% CL exclusion contours for a wino-like neutralino in the squark-gluino mass plane for the single photon analysis with the JGB method.

limits to the wino-like neutralino scenarios. In terms of $\mathrm{E}_{\mathrm{T}}^{\mathrm{miss}}$ and JGB based single photon analyses, they seem to be complementary with the JGB based more sensitive in the wino-like scenarios and thus setting tighter limits. The presented limits are the most stringest placed on these scenarios to date.

\section{References}

[1] CMS Collaboration, The CMS experiment at the CERN LHC, JINST 3 S08004 (2008).

[2] P. Meade, N. Seiberg, D. Shih, General Gauge Mediation, (2008) arXiv :0801.3278v3.

[3] P. Meade et al., General Gauge Mediation, Prog. Theor. Phys. Suppl 143 (2009) doi :110.1143/PTPS.177.143.

[4] M. Buican et al., General Gauge Mediation, European Physical Journal 016 (2006), doi :10.1088/11266708/2009/03/016.

[5] Ryuichiro Kitano, Hirosi Ooguri and Yutaka Ookouchi, Supersymmetry Breaking and Gauge Mediation, (2010), arXiv :1001.4535v2 [hep-th].

[6] S.L. Dubovsky, D.S. Gorbunov, S.V. Troitsky, Gauge mechanism of mediation of supersymmetry breaking, High Energy Physics - Phenomenology (hep-ph), doi : 10.1070/PU1999v042n07ABEH000537, arXiv :hep ph/9905466v1.

[7] CMS Collaboration, Search for Supersymmetry in Events with Photons and Missing Energy, CMS Physics Analysis Summary CMS-PAS-SUS-12-018, (2012).

[8] CMS Collaboration, SUSY Search in Photon(s) + jets $+\mathrm{E}_{\mathrm{T}}^{\mathrm{miss}}$ final state with the Jet-Gamma Balance method, CMS Physics Analysis Summary CMS-PAS-SUS-12-013, (2012).

[9] CMS Collaboration, Particle-Flow Event Reconstruction in CMS and Performance for miss Jets, Taus, and ET, CMS Physics Analysis Summary CMS-PAS-PFT09-001 (2009).

[10] CMS Collaboration, Determination of jet energy calibration and transverse momentum resolution in CMS, JINST 11 (2011).

[11] CMS Collaboration, Photon reconstruction and identification at sqrt $(s)=7 \mathrm{TeV}, \mathrm{CMS}$ Physics Analysis Summary CMS-PAS-EGM-10-005, (2010).

[12] M. Cacciari and G. Salam, Pileup subtraction using jet areas, Phys. Lett. 659 (2008) doi :10.1016/j.physletb.2007.09.077.

[13] M. Cacciari, G. Salam, and G. Soyez, The anti-kt jet clustering algorithm, Journal of High Energy Physics 04 (2008) doi :10.1088/1126-6708/2008/04/063, arXiv :0802.1189v2 [hep-ph].

[14] LHC New Physics Working Group, Simplified Models for LHC New Physics Searches, (2010). See http ://www.lhcnewphysics.org/photons.

[15] CMS Collaboration, "Search for New Physics in Events with Photons and Missing Transverse Energy at CMS", CMS Physics Analysis Summary CMS-PASSUS-12-001, (2012). 